\title{
Decoding the global outbreak of COVID-19: the nature is behind the scene
}

\author{
Piyanki Das ${ }^{1} \cdot$ Tathagata Choudhuri $^{1}$ (1)
}

Received: 20 April 2020/ Accepted: 24 May 2020/Published online: 22 June 2020

(C) Indian Virological Society 2020

\begin{abstract}
The sudden emergence of SARS-CoV-2 causing the global pandemic is a major public health concern. Though the virus is considered as a novel entity, it is not a completely new member. It is just a new version of previously emerged human SARS corona virus. The rapid evolving nature by changing host body environment and extreme environmental stability, collectively makes SARS$\mathrm{CoV}-2$ into an extremely virulent genetic variant. The evolution of the virus has been occurred by the continuous process of molecular genetic manipulation, through mutation, deletion and genetic recombinationevents. Different host body environment acts as the supportive system for the pathogen which creates extreme selective pressure. By the process of genetic evolution the pathogen developes new characters. Then the new version of the virus has been naturally selected by susceptible human host and adapt itself inside the host body causing deadly effect. Moreover, extreme environmental stability helps in the process of viral survival outside the host and its transmission. Thus both the host body or internal environment and the external environment performs equally as a source, responsible for shaping the genetic evolution of the SARSCoV-2 towards theCOVID-19 disease fitness in nature in a pandemic form.
\end{abstract}

Keywords SARS-CoV-2 - Host body environment . Molecular genetic evolution - External environment . Environment stability $\cdot$ COVID-19 pandemic

Tathagata Choudhuri

tathagata.choudhuri@visva-bharati.ac.in

1 Department of Biotechnology, Siksha-Bhavana, VisvaBharati, Santiniketan, West Bengal 731235, India

\section{Introduction}

After so many years of the discovery of DNA/RNA as the genetic base of organisms, we are not capable to fully understand the newly emerging diseases based on the genetic study. As the human population has been evolved, it is exposed to new environmental conditions in terms of migration, change in food habits and lifestyle pattern. Parallely they have interacted with wide range of new micro biome. Now in this host-pathogenic interaction, both of them are in a track for winning a race to evolve and establish itself by creating immense selective pressure over each other. Here comes the role of the key player, the nature or environment. From the origin of life, the nature ultimately selects the prevalence of the genetic makeup and decides the winner beyond the game of neucleotides. The interplay between the environment and the genetic material actually creates new or evolved genetic variety and selects its existence both for pathogen and the host and decides who will dominate in the nature $[2,17]$. This ancient story of nature's selection rule has been observed for the development of novel diseases like, small pox, cholera, tuberculosis and in recent past like, AIDS, different flus and the most related newly emerging SARS (Severe acute respiratory syndrome) and MERS (Middle East respiratory syndrome) outbreak [31].

In the current global COVID-19 outbreak, which is the major health concern in the world, it is very important to understand how this novel entity suddenly emerged and capable enough to create a global pandemic with its fetal form. In this regard prevention in form of vaccination is the only way to combat the disease and genome study is the basic thing. But beyond this, the effect of nature over it needs to be understood to evade the origin and evolution of the new genetic character and its virulent establishment 
in nature by population distribution. It has been observed that $40 \%$ of the new emerging diseases is caused by virus, and RNA virus are too much progressive in this case. Thus how these RNA based fetal pathogen with their upgraded genetic setup has been originated and selected by nature with its disease outcome must be analyzed well [5, 31]. COVID-19 is a viral disease caused by human RNA virus SARS-CoV-2. In this review we will focus on how the viral pathogen is evolved and potent enough to cause infection at pandemic level from nature's point of view. In case of infectious disease, the host-pathogen interaction must be understood where the host environment attributes a major role in the establishment of disease. In case of any pathogenic disease outcome both the host and the pathogen struggles with each other with their unique genetic setup but the gene function is not the only factor for disease establishment or the healthy body maintenance. The environmental conditions during this struggle play influential role in the action of genome, more specifically, collective action of environment and genetic mutation affects the regulation and function of genes [5]. Moreover when we consider a virus, it needs a living body to functionally active and thus we can say that, the environment for the virus to work is its host body environment. On the other hand the host body where the virus survives also needs the external environment as its survival resource during transmission. Now in the following discussion we will try to understand how these two kinds of environment interplay in the development of the virus, causing current COVID-19 outbreak.

\section{Nature is the key player of origin, evolution and pathogenicity of the virus}

To understand the root of the novel viral pathogen and role of environment for its development, we need to explain it with the molecular basis of Darwin's theory of evolution and natural selection by the continuous process of genetic permutation and combination. SARS-CoV-2 is associated with respiratory syndromes showing flue like symptoms. If we recall the emergence of different other deadly viral flue pandemics, specifically the SARS and MERS in past, all of them are of similar kind with different version and originated by species spill over and zoonotic transfer, which is occurred once in a while over a period. All of these evidences indicate towards the origin of the pathogens from a common ancestral form which needs to be investigated. The probable line of development for such pandemic outcomes happened by continuous evolutionary procedure within different species or host environment exposure, by mutation during replication or genetic recombination between two different viral species and ultimate adaptation to a susceptible host by natural selection of the new version of the viable pathogen resulting infection [7, 8]. In case of these pandemics, beyond the genetic mechanisms, varied permissive host exposure (intra or inter species spill over/intermediate host) seems an important determining factor for pathogenic evolution among human population [13, 14]. But the natural way of genetic adaptation of the current SARS-CoV-2 is not understood yet. In this part, based on its published sequence analysis we will try to figure out how the molecular genetic variation leads to viral adaptation to the human host body environment. Out of the corona virus society, the SARSCoV-2 can cause infection to humans like other human CoV. But we have to think about the fact that, its infection severity is deadly enough compared to all the other human COVID. This fact can be explained by the unique disease causing genetic character of SARS-CoV-2. Receptor recognition is the initial and important genetic determinant for a viral infection. Evidence shows that viral spike protein structural specificity decides the host range in human $\mathrm{CoV}$ like, SARS and MERS by exerting human cell receptor recognition $[14,15]$. The SARS-CoV and SARSCoV-2 both shows same binding affinity with the ACE2 (Angiotension converting enzyme 2) receptor in human cells. So it is important to understand the molecular genetic property of the spike protein receptor recognition site of SARS-CoV-2 which genetically isolates this variant from other corona virusand decides the human cell specificity and infection in more severe form. The human cell receptor binding domain of the spike protein of SARS-CoV-2 is mutated in 5-6 amino acid residues and shows high specific ACE2 human cell receptor binding affinity. The spike protein has two subunits ( $\mathrm{S} 1$ and $\mathrm{S} 2$ ) and at the junction, there is a polybasic cleavage site present which allows furin or protease mediated cleavage and insertion of prolin like molecule which adds 0-linked glycan molecules resulting the flanking of the two cleavages [2, 29]. Thus it is clear that, the uniqueness of spike protein is crucial for the genetic isolation of SARS-CoV-2 and its high affinity binding to human cells. But it has been observed from receptor homology study that, the SARS-CoV-2 also has affinity to the ACE2 receptor in other animals like the humans. Now the next question come in mind that, how SARS-CoV-2 particularly becomes pandemic for human beings with high mortality rate compared to the other one. For a specific host pathogen interaction and disease establishment, except the receptor recognition by the unique spike protein domain of the virus, the receptor variation of the host cell also is an important genetic character. The human ACE2 cell receptor is genetically identical from other animals for the recognition by the corona virus as observed for MERS-DPP4 receptor binding property [8]. Moreover it has been observed that, there are 
several ACE2 receptor variants present in different human populations and several variant out of it may restrict or reduce the $\mathrm{CoV}$ specific association with it. From the recent genetic analysis of the SARS-CoV-2 ACE2 human receptor variation in different population shows that, the expression pattern of ACE2 receptor is an important determining factor for the pathogen susceptibility. For example, among the Asians specifically, the Chinese population don't have the natural resistant mutated gene against $\mathrm{CoV}$ infection and express high level of ACE2 receptors in tissue [3]. So it is revealed that, not only the genetic uniqueness of the spike protein of pathogen, but also the receptor of host cell is equally important for the successful establishment of COVID-19 outbreak.

Now the next question is how this unique host-pathogen interactive characteristic has been developed under the influence of host environment. From different recent research evidence based on phylogenetic analysis of complete genome sequence of SARS-C0V-2 shows indication of the evolution of a rapid, diverse and sequential genome variability whichwe will analyze by molecular genetic perspective from four different angles. First we will analyses the genome variability in terms of the diversity of the SARS-CoV-2 genome in different host species. This is revealed that SARS-CoV-2 show overall $96 \%$ sequence similarity with the bat SARS-CoV like corona virus but the similarity differs in the spike protein receptor binding domain (RBD) [32, 35]. We got this kind of sequence similarity in other human corona virus where bat have been found as the common ancestral reservoir of the pathogen [10]. On the other hand, a similar kind of corona virus with SARS-CoV-2 has been identified in Malayan pangolins, which shows strong and almost identical similarity with SARS-CoV in the spike protein RBD. Thus these pangolins can be interpreted as the intermediate host for SARS-CoV2 [34]. These evidences indicate toward the sequential evolution of the spike protein RBD by species spill over nature of the virus. The variability of SARS-CoV-2 viral genome in different species confirms of its animal origin and subsequent evolution of by jumping species nature.

But none of the sequence similarity of SARS-CoV with bat or pangolins has been found based on the unique polybasic cleavage site, which can be considered as next level of determining criteria for SARS-CoV-2 human cell receptor specificity and pathogenic severity as observed in case of MERS an avian influenza virus [1, 20]. The insertion of unique poly basic furin cleavage site in SARSCoV-2 is probably acquired during human to human transmission. Thus the second indication of molecular diversity and evolution will be analyzed in terms of different subtypes of the virus, which has been found in different human population throughout the world. Most recent research work suggests three different genetically close sub types of the SARS-CoV-2 with small amino acid sequence variations which can also be linked with continental and ethnic specificity. This research shows that the A subtype is considered as the ancient form of SARS-CoV2 which shows close similarity with bat corona virus and generates the other two different subtypes, the B and C. The sub type B, which is mainly profound in China and East Asian continents is the genetic variant of the subtype A by two mutations, (synonymous and nonsynonymous) and not found outside Asia. The B subtypes has been again mutated and generated a daughter subtype the $\mathrm{C}$ variant, which dominates in Europe and represented among American populations. The C subtype differs from B by single nonsynonymous mutation [9]. This evidence of sequential development of different sub types from a single variant among different human population in different continents indicates the continuous single lineage evolution of the virus during human to human species spill over. Moreover, the different subtypes of SARS-CoV-2 can be linked with different genetic setup of the host population in the world. Now, as previously described, different SARSCoV-2 infected population in the world shows genome variation and expression of ACE2 receptor coding sequence which can affect the binding and susceptibility of the SARS-CoV-2, it can be predicted that, this ACE2 receptor variation created intense selective pressure to the circulating SARS-CoV-2 genome and as a result of this the virus adapted to establish infection with its different genetic variants in a pandemic form throughout the world [3].

Every disease when it becomes a pandemic it is revealed sequentially through several stages, which also indicates toward the evolutionary nature of the pathogen with the host body. So the third molecular genetic evolution of the COVID-19 will be analysis based on the different phages of the pandemic. If we categories the COVID-19 outbreak into different stages, it has been found that at the initial stage it is reported in some individuals in Hunan market of Wuhan, in Hubei province of China. In the second phase, it has started to found as clustered form in different regions of China. Then in the third stage, the disease has been disseminated in different parts of Europe, America and other Asian countries and resulted as a pandemic. Now this different phase of disease progression COVID-19 outbreak till now, can be linked with the evolutionary pattern of SARS-CoV-2 and its different subtypes (as previously described the A,B,C) emerged in different population of the world. The first emergence and case reports of SARSCoV-2 is found in China which represents the B subtype genome setup and specific to this region only. The B type is reported to be originated from the ancestral betacorona virus showing close sequence similarity. The association of the virus to the live stock animal market in China supports 
this origin [35]. Now the pangolin theory as intermediate host for the development of the unique receptor binding site (RBD) in the SARS-CoV-2 can be linked with the report of SARS corona virus in dead pangolins from Guangdong wild life resource in China during the period of COVID outbreak in China [34]. This B subtype with the evolved genetic setup caused clustered infection in different parts of south Asia soon after it infected a large population inside China. Later, after a short lag period, the virus is mutated and emerged as a pandemic in different parts outside Asia with another variant, the $\mathrm{C}$ subtype, profound in European and American countries. This step indicates another stage of variation with probable acquisition of the polybasic cleavage site under the influence of unique ACE2 receptor specificity in these population. Thus it is clear that the viral lineage of SARS-CoV-2 is constantly associated with evolutionary process that leads to the generation of different subtypes of the same species which is gradually revealed through different phases of the disease and spreaded among different population of the world causing pandemic.

Evidence form our fourth and last analysis, it has been observed that mutation of the viral genome and genetic recombination is another process which confirms the molecular genetic variability of the pathogen. Several mutation and deletion of SARS-CoV-2 genome has been observed from different SARS-CoV-2 genome sequence references. It has been observed that mutation has been occurred in the different structural and non-structural glycoproteins like 5' ORFlabpolyprotein, envelop protein, matrix protein and most important determining factor the viral spike surface glycoprotein in RBD domain. On this note, no such change has been observed in the envelope protein. Such type of mutation has been also observed in betacorona viruses. Not only genetic mutation or deletion, events of genetic recombination has been observed in case of origin of SARS-CoV-2 spike protein RBD. Genetic recombination events among bat, pangolin and human corona virus confirms this $[16,21,26]$.

So it is clear from the above study that, generation of new versions of viral diseases in humans is acquired due to the continuous molecular genetic operations inside host body by continuous species transmission. This event can be supported by the evidence of acquisition of adapted character during repeated cell culture passagingof the SARS-CoV [23]. But on this note it should be pointed that, new disease may appear at any time, in any places, among any population which is unpredictable, because evolution is a collective action of genetic operation and environmental effect. In case of virus the host body is the key factor which actually gives this opportunity or favorable environment for these genetic operations to happen because the virus needs a host body as a resource to be functionally active. It is the normal tendency of a microbial species to find a convincible way to transmit and adapt itself to new environment for evolution. In case of virus, more specifically for the RNA virus, the tendency is high where there is continuous circulation of the viral genome among different host species [28]. During this period, host environment creates intense selective pressure to the pathogen. The host body environment is acting as an influential factor for the virus to take a do or die decission. When the virusis successful,it is naturally selected and adapted to establish its character by gettingthe control over the host's genetic construction by exerting virulent effects. When this process continuously goes on by exposure to new environments, it can lead to an extent where it can cause a pandemic, otherwise it is the end of line for the pathogen to evolve. This is a gradual process, when the virus accumulate the required genetic makeup or prepare itself and takes several attempt for the big jump. During this preparatory phase the pathogen can generate mild infection or infection to a limited bunch of population or create silent carriers depending on the host body environment. These carriers don't show any symptoms but the infectious pathogen with its new genetic makeup is maintained here and can be transmitted to different suitable host environment for disease establishment. Thus these carriers are very crucial, and might be another strategy of the virus for the evolution of new genetic setup. An evolved character is developed under environmental pressure [25]. In this case, the protective immune system of the host body environment helps the virus to evolve under selective pressure of infection rejection. Identification of several asymptomatic carriers for SARS-CoV-2 can be a result of the above fact. Thus virus is the player, but its' optimum molecular genetic fitness, distribution and evolution are shaped by the host body environment [12, 19, 24]. In case of SARS-CoV-2 its diverse genetic variability and evidence of its existence among different host species indicates towards its rapid "jumping species" nature which probably makes it extremely diverse and virulent. The host body environment gave the virus the opportunity to prepare for the battle where both the genetic manipulation and the natural selection process plays major role for its adaptability resulting the current uncontrolled pandemic.

From another angle if we look over the two extremities of the origin and exposure of the SARS-CoV-2, they are bats and humans. Both of the host environments can be considered as large population. Bats is the reservoir of a large number of viral pathogens and inhabit in a giggling manner in a close contact thus enhances the chance of cross transmission and giving the pathogen a field to evolve. Similarly, the modern human population is highly dynamic and exposed to various extreme environmental conditions. The large co habitation and lifestyle pattern enhances the 
chance of cross transmission of virus and make a susceptible environment for emerging new viral disease.

\section{Nature helps the viral stability and survival outside the host}

After considering the impact of molecular genetic diversity and host body environment in the origin and development of a virulent strain, we need to understand the role of the external environmental factors in the stability and survival of the virus outside the host body conditions. During the process of evolution, intra or inter species transmission among the susceptible host community, external environment plays a critical role. Though in the external environment, outside the host body, the virus cannot multiply and cause infection but different factors are crucial in the stability of the virus outside its host environment for the process of its transmission. Specifically when we are dealing with a respiratory tract associated virus, the role of environmental factors must be emphasized because the respiratory outputs are directly exposed to the environment through cough, sneeze, and sputum. The role of different environmental factors like temperature, salinity, $\mathrm{P}^{\mathrm{H}}$ etc. for viral stability in aerosol, inert surface, water etc. needs to be discussed, because external environment is the connecting media between various host species during the viral transmission. In case of the other two common human corona virus super spread, SARS-CoV-1 and MERS air transmission is well reported in form of droplet and aerosol.

The evolutionary pattern of SARS-CoV-2 is associated with geographical continent specific subtype diversity with a tendency of rapid species spill over which indicate towards its huge external environmental adaptation. Moreover social distancing is emphasized for COVID-19 spread due to its highly contagious nature. Thus understanding the role of external environmental factors in maintaining viral stability and capability to survive in different patient samples as well as certain inert or abiotic environmental substances, the fomite, by which it can spread needs to be analyzed very well. If we consider the clinical samples by which the virus is exposed to the environment, research shows that SARS-CoV-2 may present in different types of patient samples. High viral load is found mainly in the respiratory samples. Presence of virus in blood samples proves its systemic spread. Detection of live viral particle in feces indicates its fecal-oral route of environmental exposure $[6,30]$. After the virus is exposed to environment by these clinical samples, SARSCoV-2 shows various degree of fomite stability for different surfaces associated to those patient samples. In case of steel surface, the stability is maximum (approximately
2-3 days). It is also stable in polypropylene, card board and copper (show less degree of stability). In aerosol the virus shows three hours of stability [27]. In connection with the fecal-oral route the stability and sustainability of SARS$\mathrm{CoV}-2$ has been found in waste and sanitary water in infected area,is recently reported [18].

The stability of virus in different contaminated surfaces and in different patient sample outputs depends on the action of temperature and relative humidity percentage. It has been observed for corona virus that low temperature (4 degree) and low humidity (20\%) is supportive for the viral stability, where gradual increase of both the parameters inactivates the virus more rapidly. It has been observed that lipid virus like COVID can be inactivated by the interference of two mechanisms, the air water interphase and desiccation process. When the relative humidity is nearly $50 \%$, the virus is accumulated by the air water interphase. Then the desiccation process excretes water from the lipid layer by oxidation and simultaneously breaks the cross linking thus which effectively damage the lipid layer of the pathogen much rapidly [4]. On this note it must be pointed that mucus acts as the first protective barrier for invading respiratory virus. Interaction of mucus layer glycoprotein with viral surface proteins have been well reported in influenza virus. Recent research shows that the saliva or mucus substance may act as a protective barrier for the virus when it is exposed to the external environment. The aerosol also goes with the same flow, where it helps the virus from the hazardous effect of varying external environment by retaining its infectivity [11,33]. So temperature inactivation or the air water desiccation mechanism, are not effective on the virus when the organic barriers acts as insulator.

Thus in case of SARS-COV-2, it is received by the environment through different clinical sample outputs mainly the respiratory secretions from an infected person whether it is a susceptible host or carrier, in form of droplets or aerosol and can survive in this form in various fomites. After the virus is received in the external environment, the patient sample itself protects it from adverse environmental conditions and helps it to survive or sustain for long time acting as maintaining media for the virus outside the host body. This is the probable reason why SARS-CoV-2 shows high degree of external environment stability. From the above analysis it is clear that the outside host environment acts as the intermediate player which receives, maintains, protects and transfers the pathogen using its various factors as weapon for COVID-19 pandemic. 


\section{Concluding remarks}

From the above study it is clear that rapid evolving nature and high environmental stability of SARS-CoV-2 is responsible for the current outbreak of COVID-19. The evolutionary process of this new version of the virus indicates the 'synthetic theory of evolution' or the 'NeoDarwinism theory' where, the virus has gone through sequential multistep thorough process of molecular genetic variation which is shaped and established by the natural selection process. In this review the role of both the host body environment and the external environment in disease establishment is discussed from different angle (Fig. 1).

The host body is the important factor for the COVID-19 host-pathogenic interaction establishment where the molecular evolution happens. The rapid host body changing pattern helps the virus to be continuously exposed to new environmental conditions, which creates selective pressure to the virus. In order to struggle for existence and infection establishment inside host body, the virus undergo through several genetic manipulations and achieve its mutated characters. Then genetically close different subtypes of SARS-CoV-2 develops unique spike protein receptor binding domain with high degree of receptor binding property to human cells and adapt itself to fit the character inside the host body. Ultimately by the action of natural selection, specific host-pathogen interaction happens where the virus can establish the infection.

The external environment acts as intermediate player in between one host to another, by providing suitable environment for stability and survival for SARS-CoV-2 outside its host body resulting high viability and higher transmissibility compared to other corona virus. It has been reported that the external environment also interacts with the host body environment and helps the stability of the virus inside the host body system and its infectivity. Changing temperature and relative humidity contributes to the degree of host susceptibility to a certain pathogen, pathogen infiltration and survival inside the host body [22] Thus the outcomes from the whole study will help to understand the sudden emergence of COVID-19 pandemic and its character. The findings emphasize to consider the interactive role of both of the two environments, the host body environment and the external environment as the source of genetic evolution of emerging viral diseases and its control.

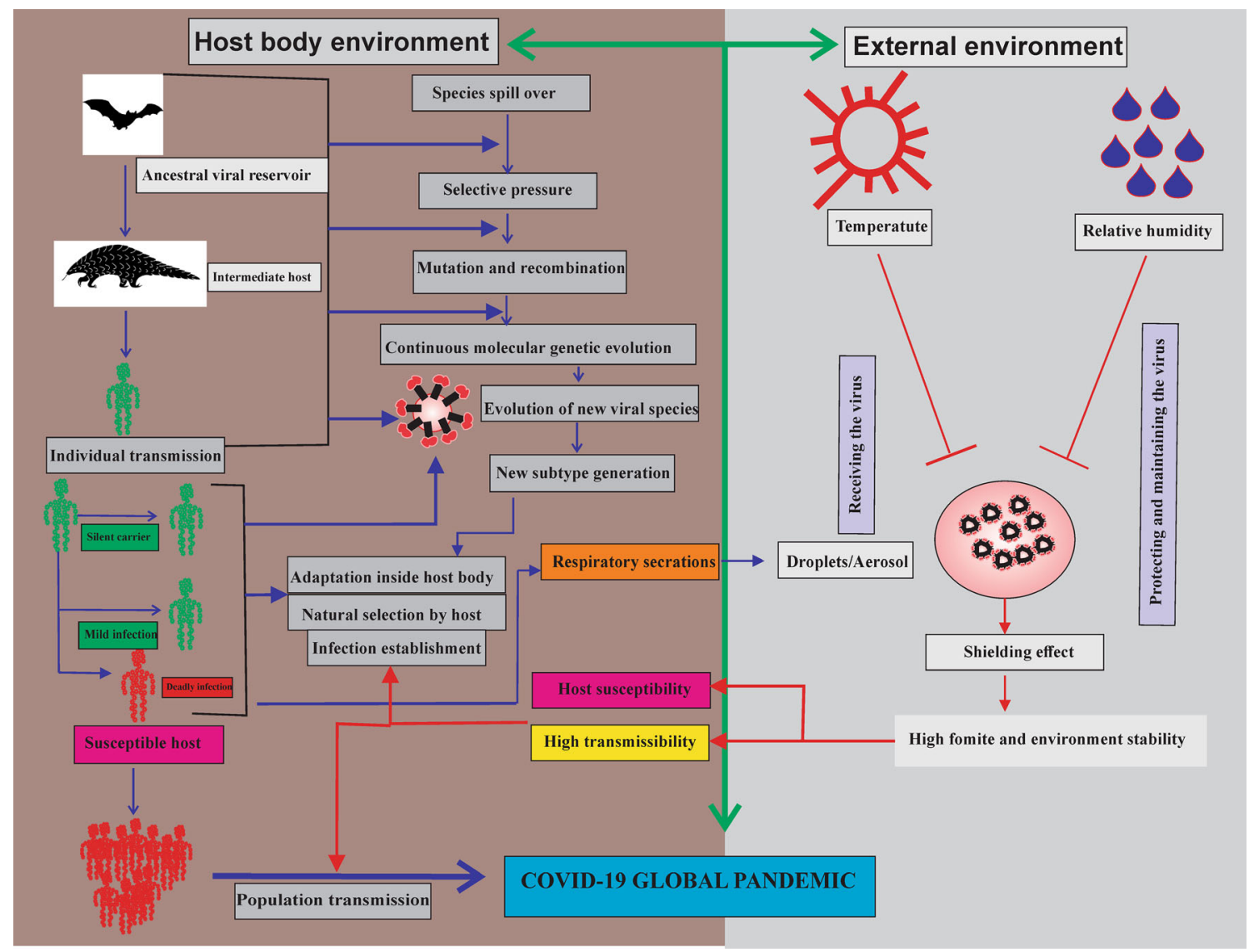

Fig. 1 Interactive role of host body environment and external environment in the molecular genetic evolution of SARS-CoV-2 and COVID-19 pandemic 
Acknowledgements We would like to thank Mr. Laltu Hazra, regarding his general laboratory help and CSIR for their financial assistance by CSIR SRF fellowship for PD (Council of Scientific and Industrial Research, Govt. of India, Senior Research Fellowship). TC is a Fellow of Indian Virological Society. The authors also thank IIT Delhi HPC facility for computational resources.

\section{Compliance with ethical standards}

Conflict of interest The authors declare that they have no conflict of interest.

\section{References}

1. Alexander DJ, Brown IH. History of highly pathogenic avian influenza. Revue scientifique et technique (International Office of Epizootics). 2009;28(1):19-38.

2. Andersen KG, Rambaut A, Lipkin WI, Holmes EC, Garry RF. The proximal origin of SARS-CoV-2. Nat Med. 2020;26(4):450-2.

3. Cao Y, Li L, Feng Z, Wan S, Huang P, Sun X, et al. Comparative genetic analysis of the novel coronavirus (2019-nCoV/SARS$\mathrm{CoV}-2)$ receptor ACE2 in different populations. Cell Discov. 2020;6(1):11.

4. Casanova L, Jeon S, Rutala W, Weber D, Sobsey M. Effects of air temperature and relative humidity on coronavirus survival on surfaces. Appl Environ Microbiol. 2010;76:2712-7.

5. Chakravarti A, Little P. Nature, nurture and human disease. Nature. 2003;421:412-4.

6. Chen Y, Chen L, Deng Q, Zhang G, Wu K, Ni L, et al. The presence of SARS-CoV-2 RNA in feces of COVID-19 patients. J Med Virol. 2020;92(7):833-40.

7. Chinese SARS Molecular Epidemiology Consortium. Molecular evolution of the SARS coronavirus during the course of the SARS epidemic in China. Science. 2004;303(5664):1666-9.

8. Cui J, Li F, Shi Z-L. Origin and evolution of pathogenic coronaviruses. Nat Rev Microbiol. 2019;17(3):181-92.

9. Forster P, Forster L, Renfrew C, Forster M. Phylogenetic network analysis of SARS-CoV-2 genomes. Proc Natl Acad Sci. 2020;117(17):9241-3.

10. Hu B, Ge X, Wang L-F, Shi Z. Bat origin of human coronaviruses. Virol J. 2015;12(1):221.

11. Kormuth KA, Lin K, Prussin AJ 2nd, Vejerano EP, Tiwari AJ, Cox SS, et al. Influenza virus infectivity is retained in aerosols and droplets independent of relative humidity. J Infect Dis. 2018;218(5):739-47.

12. Lalić J, Cuevas JM, Elena SF. Effect of host species on the distribution of mutational fitness effects for an RNA virus. PLoS Genet. 2011;7(11):e1002378.

13. Lau S, Wong A, Lau T, Woo P. Molecular evolution of MERS coronavirus: dromedaries as a recent intermediate host or longtime animal reservoir? Int J Mol Sci. 2017;18:2138.

14. Letko M, Miazgowicz K, McMinn R, Seifert S, Sola I, Enjuanes $\mathrm{L}$, et al. Adaptive evolution of MERS-CoV to species variation in DPP4. Cell Rep. 2018;24:1730-7.

15. Li F. Receptor recognition mechanisms of coronaviruses: a decade of structural studies. J Virol. 2015;89(4):1954-64.
16. Li X, Giorgi E, Marichann M, Foley B, Xiao C, Kong X-P, et al. Emergence of SARS-CoV-2 through recombination and strong purifying selection. 2020.

17. Lindahl JF, Grace D. The consequences of human actions on risks for infectious diseases: a review. Infect Ecol Epidemiol. 2015;5(1):30048.

18. Lodder W, de Roda Husman AM. SARS-CoV-2 in wastewater: potential health risk, but also data source. Lancet Gastroenterol Hepatol. 2020;5(6):533-4.

19. Makeyev EV, Bamford DH. Evolutionary potential of an RNA virus. J Virol. 2004;78(4):2114-20.

20. Menachery VD, Dinnon KH, Yount BL, McAnarney ET, Gralinski LE, Hale A, et al. Trypsin treatment unlocks barrier for zoonotic bat coronavirus infection. J Virol. 2020;94(5):e01774-19.

21. Phan T. Genetic diversity and evolution of SARS-CoV-2. Infect Genet Evol. 2020;81:104260.

22. Roberts KE, Hadfield JD, Sharma MD, Longdon B. Changes in temperature alter the potential outcomes of virus host shifts. PLoS Pathog. 2018;14(10):e1007185.

23. Sheahan T, Rockx B, Donaldson E, Corti D, Baric R. Pathways of cross-species transmission of synthetically reconstructed zoonotic severe acute respiratory syndrome coronavirus. J Virol. 2008;82(17):8721.

24. Slingenbergh J. Animal virus ecology and evolution are shaped by the virus host-body infiltration and colonization pattern. bioRxiv. 2018:492603.

25. Sorrell I, White A, Pedersen AB, Hails RS, Boots M. The evolution of covert, silent infection as a parasite strategy. Proc Biol Sci. 2009;276(1665):2217-26.

26. Su S, Wong G, Shi W, Liu J, Lai ACK, Zhou J, et al. Epidemiology, genetic recombination, and pathogenesis of coronaviruses. Trends Microbiol. 2016;24(6):490-502.

27. van Doremalen N, Bushmaker T, Morris DH, Holbrook MG, Gamble A, Williamson BN, et al. Aerosol and surface stability of SARS-CoV-2 as compared with SARS-CoV-1. New Engl J Med. 2020;382(16):1564-7.

28. Viruses switch hosts to evolve. Nature. 2017;543(7646):466.

29. Walls AC, Park Y-J, Tortorici MA, Wall A, McGuire AT, Veesler D. Structure, function, and antigenicity of the SARSCoV-2 spike glycoprotein. Cell. 2020;181(2):281-92.e6.

30. Wang W, Xu Y, Gao R, Lu R, Han K, Wu G, et al. Detection of SARS-CoV-2 in different types of clinical specimens. JAMA. 2020.

31. Weiss R, McMichael A. Social and environmental risk factors in the emergence of infectious diseases. Nat Med. 2005;10:S70-6.

32. Wu F, Zhao S, Yu B, Chen Y-M, Wang W, Song Z-G, et al. A new coronavirus associated with human respiratory disease in China. Nature. 2020;579(7798):265-9.

33. Zanin M, Baviskar P, Webster R, Webby R. The interaction between respiratory pathogens and mucus. Cell Host Microbe. 2016;19(2):159-68.

34. Zhang T, Wu Q, Zhang Z. Pangolin homology associated with 2019-nCoV. bioRxiv. 2020:2020.02.19.950253.

35. Zhou P, Yang X-L, Wang X-G, Hu B, Zhang L, Zhang W, et al. A pneumonia outbreak associated with a new coronavirus of probable bat origin. Nature. 2020;579(7798):270-3.

Publisher's Note Springer Nature remains neutral with regard to jurisdictional claims in published maps and institutional affiliations. 